\title{
Surgical and haemodynamic considerations in middle aortic syndrome
}

\author{
SAIBAL GUPTA \\ From the Department of Cardiothoracic Surgery, NRS Medical College, Calcutta 700014, India
}

ABSTRACT Eleven patients with the middle aortic syndrome have been investigated during the past five years. Corrective surgery was possible in seven, five of whom survived. The maximum constriction in most cases was at the thoracoabdominal junction, an area normally surrounded by intestinal lymphatics. This may indicate that the aetiology is related to an immune response to parasitic infestation of the intestines. Comparison of the extent and severity of the disease as found at operation with the results of preoperative investigations such as angiography, radioisotope renography, and measurement of angiotensin in the renal veins showed that in some patients surgical exploration was more accurate in deciding the feasibility and the method of surgical correction.

Inflammatory occlusion of the branches of the aortic arch was reported by Broadbent (1875) and subsequently described by Takayasu (1908). Similar affections of the descending thoracic and abdominal aorta were described much later, and the similarity between this condition and congenital coarctation of the aorta in clinical features and presentation was recognised (Shikhare, 1921; Bahnson et al, 1949; Konar et al, 1955; Basu, 1961; Sen et al, 1962). This condition has also been given the name of "middle aortic syndrome" (Sen et al, 1963). But whereas the clinical picture and the pathology have been well recorded, publications on the surgical management of the condition are scanty (DeBakey et al, 1958; Hallman et al, 1966). This paper describes experiences with surgical reconstruction in a series of 11 patients presenting with middle aortic syndrome over the past five years.

\section{Patients and results}

There were ten female patients between the ages of 8 and 40 years and a boy aged 10 . Out of 11 patients in this series, surgical reconstruction was attempted in nine and could be completed in seven, of whom five survived.

PATIENTS UNSUITABLE FOR RECONSTRUCTIVE SURGERY

The four patients in whom surgical reconstruction could not be completed are described below. They were all women over 30 .

Case 1-A 40-year-old woman complained of continuous headache and giddiness with backache, generalised weakness, and dyspnoea on exertion. Pulses were absent from all four limbs and the left carotid artery. The right carotid pulse was feeble. There was a bruit in the upper abdomen. Angiography showed total occlusion of the right and left subclavian and left common carotid arteries and a partial occlusion of the right 3 . common carotid artery. The descending thoracic and abdominal aorta were diffusely narrowed with maximum constriction at the thoracoabdominal o junction and very narrow renal arteries on both sides. Surgical reconstruction was not attempted.

Case 2-A 30-year-old woman presented with pain in the chest, dyspnoea, and palpitations on $\sigma$ exertion. She was hypertensive with a blowing $N$ systolic bruit in the back along the course of the N aorta. Chest radiographs showed a wide medi- 0 astinum. Angiography showed a normal ascending aorta and aortic arch and branches. There was a

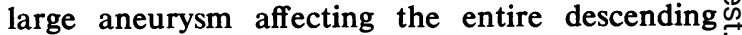
thoracic aorta. Below the aneurysm the aorta at 0 the thoracoabdominal junction was almost completely occluded. Angiography of the abdominal aorta showed another aneurysm below the co- $\frac{P}{\Phi}$ arcted segment affecting the origins of the coeliac $\frac{}{\sigma}$ and superior mesenteric arteries, but the two renal arteries appeared to be normal (fig 1). The 


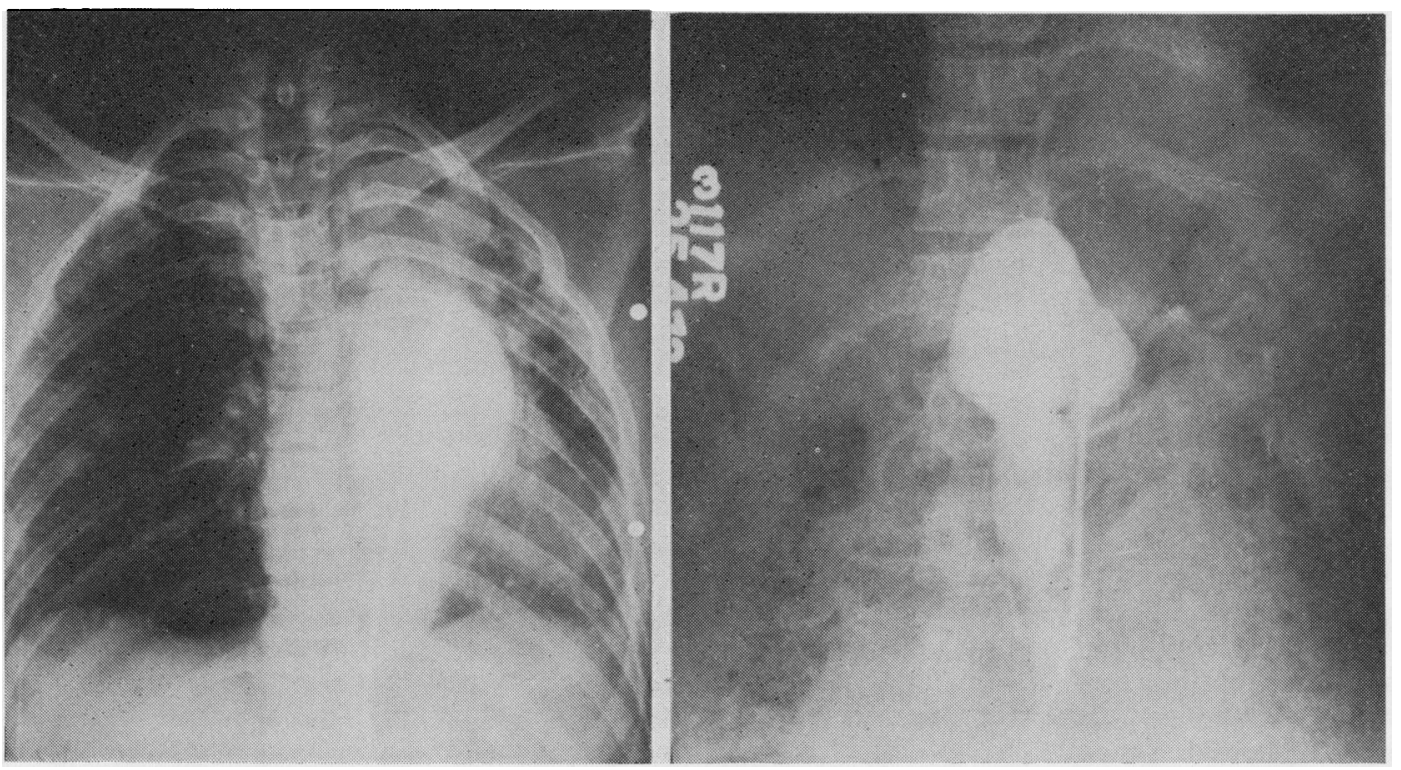

Fig 1 Case 2. Preoperative descending thoracic and abdominal aortogram. (See text for description.)

patient was operated on with partial left heart bypass using cannulae in the left atrium and left femoral artery. The thoracic aneurysm was first excised, and the upper end of a preclotted Dacron graft was attached to the aorta immediately distal to the left subclavian artery. The abdominal aorta was next exposed. The entire abdominal aorta was found to have more than one irregular lumen, as a result of chronic dissection, secondary sacs, and multiple coarctations. The whole vascular maze was enveloped in thick, congested fibrous tissue. Sterile vascular grafts of different types necessary to make a vessel by vessel repair in such a situation were not available. A less satisfactory repair was done by division of septa and plication of abnormal channels within the diseased aorta. The lower end of the graft was anastomosed to the repaired aorta just above the origin of the coeliac artery. These dissections in a heparinised patient produced a massive blood loss that could not be replaced. The heart went into permanent asystole a few minutes after releasing the clamps. In retrospect it was obvious that we had underestimated the severity of the disease from the angiographic appearance.

Case 3-A woman aged 36 presented with dyspnoea and palpitations on exertion. She was hypertensive with absence of arterial pulses in both legs. Angiography showed a diffuse narrowing of the lower thoracic and abdominal aorta but the lower abdominal aorta and the iliac arteries were not properly visualised. The abdomen was explored. The lower abdominal aorta and both common iliac and external iliac arteries were diffusely diseased. The attempt at reconstruction was abandoned.

Case 4-A 35-year-old woman presented with severe dyspnoea on the slightest exertion. She was found to have severe aortic regurgitation with left ventricular failure and absence of the pulses in both feet. Ten years earlier she had been operated on in another hospital with the diagnosis of coarctation of the aorta but a diffuse narrowing of the thoracic aorta was found and no surgical procedure was carried out. Angiography confirmed the diagnosis of severe aortic regurgitation with middle aortic syndrome but no surgical treatment was possible because of the terminal cardiac failure.

\section{PATIENTS TREATED SURGICALLY}

The seven patients in whom surgical reconstruction could be completed were as follows.

Case 5-An eight-year-old girl presented with dyspnoea and palpitation on exertion of four months' duration, hypertension, and absence of the arterial pulses in both legs. The aortogram showed an obstruction in the aorta immediately beyond the origin of the left subclavian artery. She was operated on with a diagnosis of congenital coarctation of the aorta but was found to have a $5 \mathrm{~cm}$ long inflammatory stricture. A $10 \mathrm{~cm}$ long 
segment of descending thoracic aorta was excised and replaced by a Dacron graft. She recovered after a stormy postoperative period, and her arterial pressure returned to normal. She was lost to follow-up a year after operation.

Case 6-A girl aged 19 presented with dyspnoea and palpitations on exertion, hypertension, and feeble femoral pulses. Angiography showed a coarctation of the aorta at the thoracoabdominal junction, fairly localised without involvement of the abdominal branches. Through a left thoracoabdominal incision a Dacron bypass graft was inserted between the lower thoracic aorta and the lower abdominal aorta. She made an uneventful recovery, and her blood pressure returned to normal.

Case 7-A girl aged 17 presented with a residual right-sided hemiparesis resulting from a cerebrovascular accident three months earlier. She also complained of dyspnoea and palpitations on exertion. Preliminary investigations at another hospital showed systemic hypertension as the cause of the cerebrovascular episode. Angiography showed a diffuse narrowing of the descending thoracic aorta with maximum constriction at the thoracoabdominal junction. The branches of the aortic arch were normal, there was a stenosis at the origin of the left renal artery, and the abdominal aorta was normal (fig 2). Radioisotope renography showed depressed circulation in both kidneys, more pronounced on the left side. At $\stackrel{\overrightarrow{\bar{N}}}{\frac{\vec{O}}{O}}$ operation a Dacron bypass graft was inserted between the lower thoracic and the lower abdominal aorta through a single left thoracoabdominal incision extending along the midline to the lower abdomen. The left renal artery was dissected for is a secondary bypass graft but was found to be $\overrightarrow{0}$ encircled by a fibrous collar at its origin. This was $\overrightarrow{\vec{\omega}}$ divided, which restored the arterial lumen to $\vec{\omega}$ normal and obliterated the pressure gradient $\vec{F}$ across the stenosed segment. The patient made an uneventful recovery and her arterial pressure re- $\stackrel{\omega}{\perp}$ turned to normal, and has remained so. An angiogram (fig 3) a year after operation showed the functioning bypass graft and a normal left renal artery.

Case $8-\mathrm{A}$ boy aged 10 years presented with $\vec{\nabla}$ dyspnoea and palpitations on exertion and throbbing headache of one month's duration. He was hypertensive with early notching of the ribs on the chest radiograph. Angiography showed a short narrow segment of the aorta immediately beyond the origin of the left subclavian artery, which could have been either an atypical congenital or an acquired coarctation (fig 4). At operation a $5 \mathrm{~cm}$ long inflamed fibrosed aorta was seen about $2.5 \mathrm{~cm}$ distal to the left subclavian artery. It was $\stackrel{\varnothing}{\varrho}$ bypassed by a large sized Dacron graft anas- $\overrightarrow{\overrightarrow{0}}$ tomosed well beyond the diseased area. While the $\exists$ dissection was in progress cyanosis occurred from

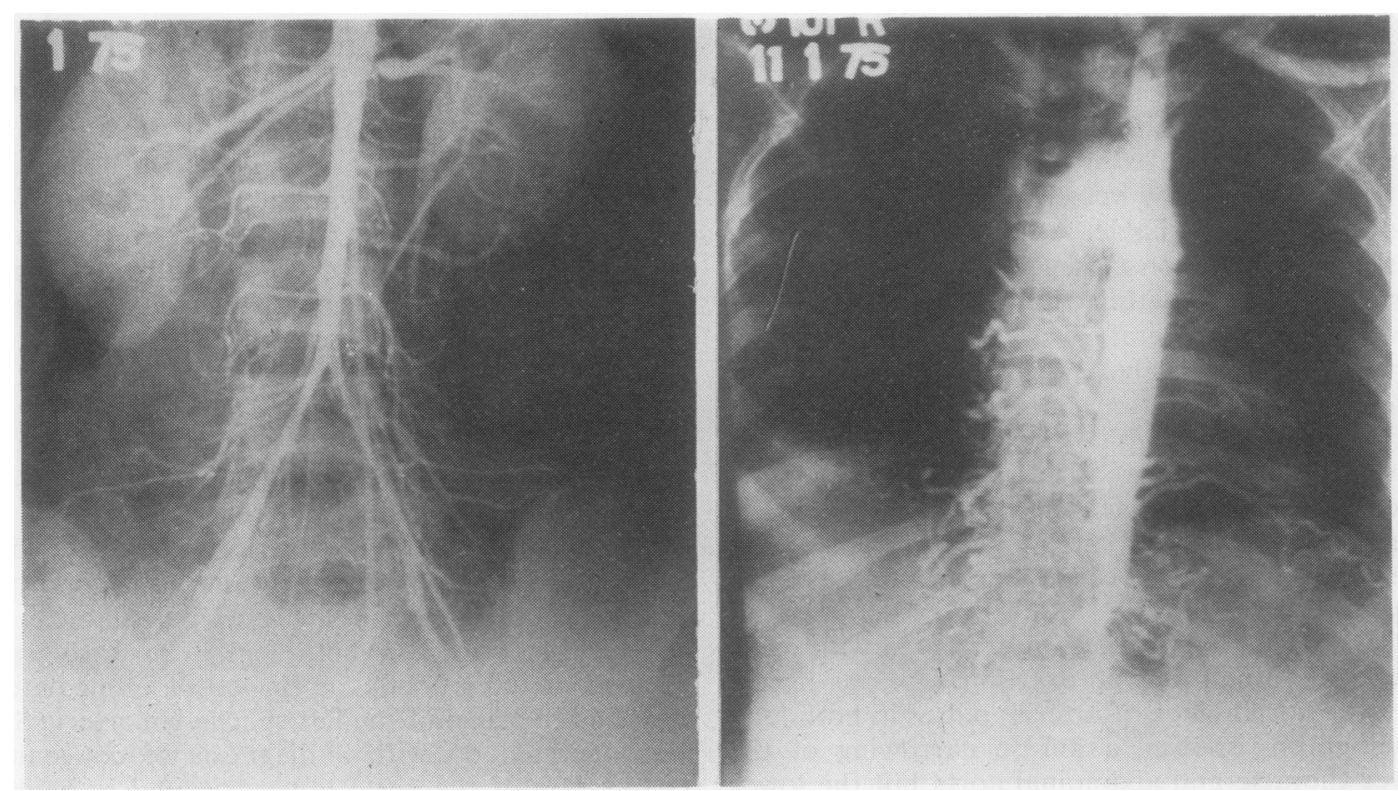

Fig 2 Case 7. Preoperative descending thoracic and abdominal aortogram. (See text for description.) 


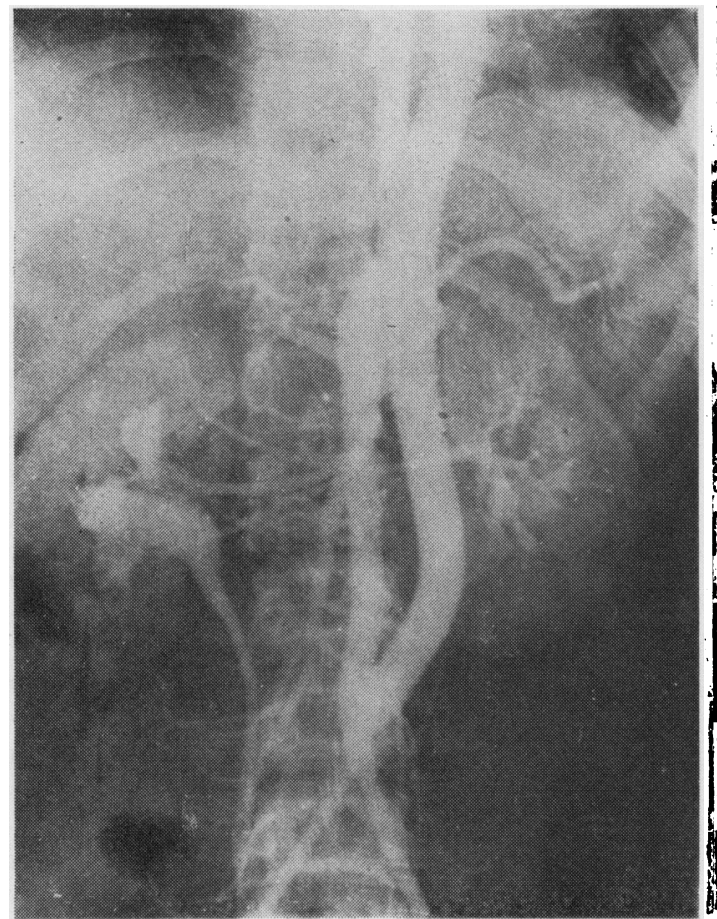

Fig 3 Case 7. Postoperative abdominal aortogram. (See text for description.) some respiratory obstruction that could not be reversed completely. During the anastomosis the heart stopped, but there was some delay in detecting the arrest. Cardiac action was restored, and the operation completed, but the child had sustained irreversible cerebral damage and died after a few days.

Case 9-A girl aged 16 years presented with dyspnoea on exertion, bleeding from the gums, and intermittent claudication in both legs. The symptoms had started about six years earlier. She was hypertensive with a systolic murmur in the aortic area and a bruit over the abdomen. Arterial pulses were absent from both legs except for a feeble pulse in the right femoral artery. Angiography showed a diffuse narrowing of the lower thoracic aorta that became almost complete in the upper adbomen. The coeliac axis, the superior mesenteric artery, and both renal arteries were visualised but the lower abdominal aorta was not opacified. There was a partial block in the superior mesenteric artery somewhat distal to its origin (fig 5) that was not noticed before operation. The patient had no symptoms of intestinal ischaemia. At operation the abdomen was explored first. The lower abdominal aorta for a distance of $5 \mathrm{~cm}$ above the bifurcation was found to be healthy and suitable for anastomosis of the graft. The chest
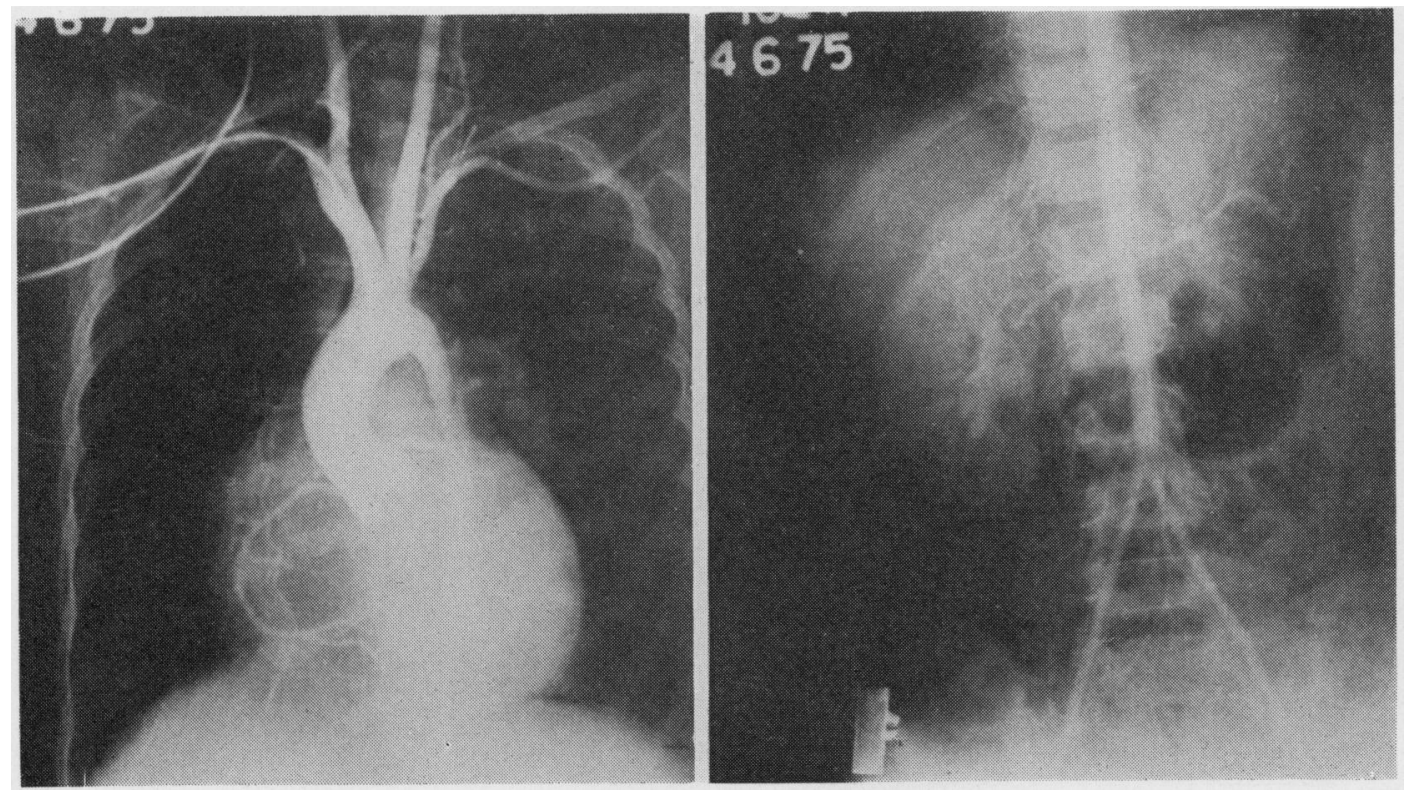

Fig 4 Case 8. Preoperative aortogram. (See text for description.) 


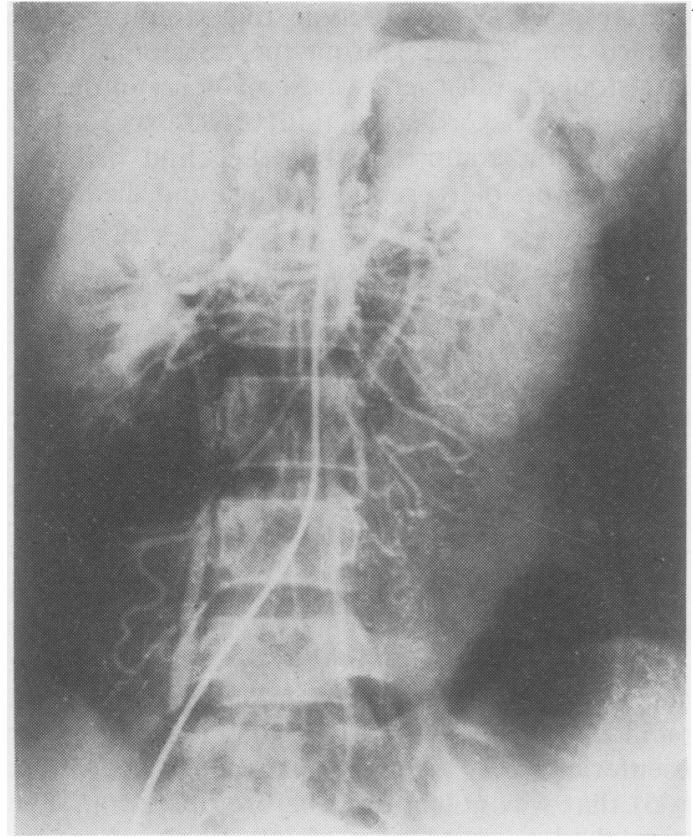

Fig 5 Case 9. Preoperative abdominal aortogram. (See text for description.) was opened next, and a long bypass graft was inserted from the upper thoracic to the lower abdominal aorta. No other branches were bypassed since the two mesenteric arteries and the two renal arteries had appeared to be normal on the angiogram. The arterial pressure returned to normal, and her recovery was satisfactory apart from a persistent tachycardia. On the second day after operation she developed distension of the abdomen with absence of bowel sounds. This was thought to be due to paralytic ileus until she became very toxic on the third day and died on the fourth. Necropsy showed gangrene of the whole of the small intestine. The lesion in the superior mesenteric artery was then detected and confirmed on the angiogram retrospectively.

Case 10-A 21-year-old woman presented with dyspnoea and palpitations on slight exertion, throbbing headache, and intermittent claudication for two years. She was hypertensive with very feeble arterial pulses in the feet and a bruit in the abdomen. Angiography showed a diffuse narrowing of the entire descending thoracic aorta with maximum constriction at the thoracolumbar junction (fig 6). The abdominal aorta and all its branches were normal. Radioisotope renography

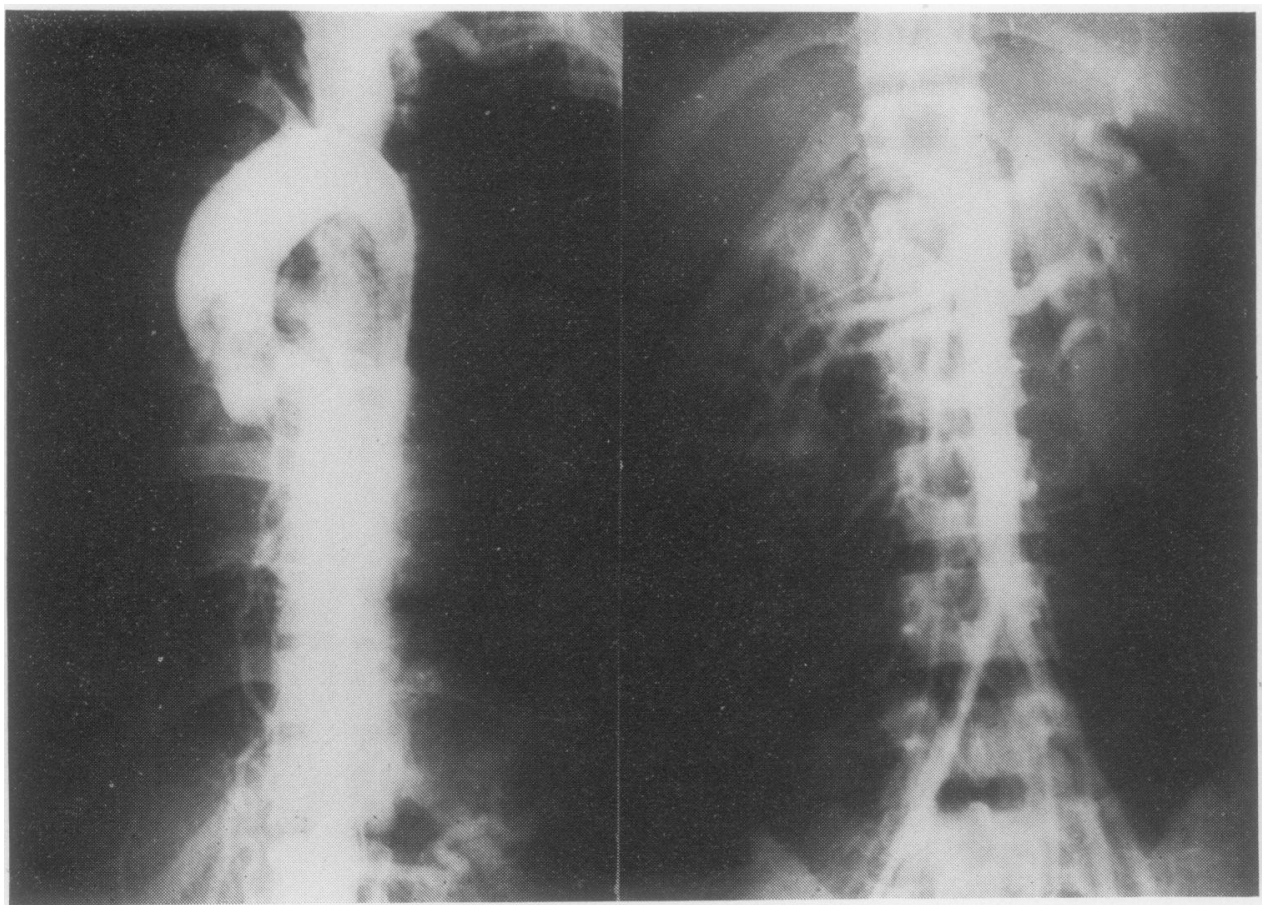

Fig 6 Case 10. Preoperative aortogram. (See text for description.) 
showed equally depressed blood flow in both kidneys. At operation a Dacron bypass graft was inserted between the distal part of the arch of aorta and the lower abdominal aorta. The patient made an uneventful recovery, and her blood pressure has remained normal. Radioisotope renography more than one year after operation showed normal blood flow in both kidneys.

Case 11-A woman aged 35 presented with vertigo, inability to concentrate, and palpitations for two and a half years. She was thin with severe hypertension and absence of arterial pulsation in the feet except for a feeble pulse in the right femoral artery. There was a bruit in the abdomen. Angiography showed a diffuse narrowing of the descending thoracic aorta with almost total occlusion at the thoracolumbar junction and upper abdominal aorta, affecting the origins of the coeliac and superior mesenteric arteries. The interrenal aorta and origins of both renal arteries were grossly distorted (fig 7). The lowest part of abdominal aorta and the inferior mesenteric artery were normal with a large upper left colic branch providing collateral supplies to the intestine. The problem in this case was to decide whether the renal arteries needed grafting. It was decided from our previous experience in case 9 that the superior mesenteric artery needed grafting though the patient denied symptoms of intestinal ischaemia. Radioisotope renography showed considerably de-

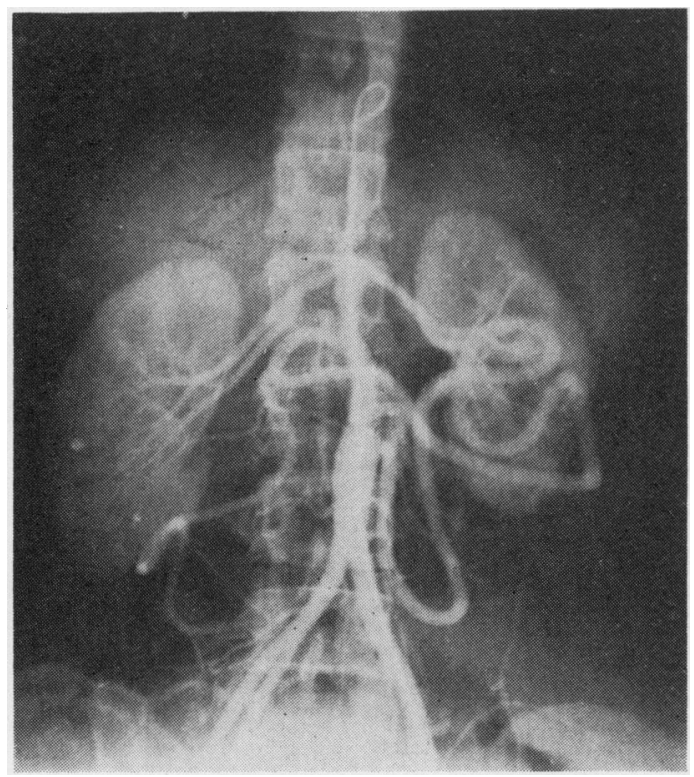

Fig. 7 Case 11. Preoperative abdominal aortogram. (See text for description.) pressed blood flow in both kidneys (fig 8). According to our experience in case 10 this could be explained by the low aortic stricture without necessarily pointing to an intrinsic renal artery lesion. Rehal vein catheterisation was performed, and the angiotensin activity in the blood samples from both renal veins and the infrarenal vena cava were measured by the bioassay methods of Regoli and Vane (1964). This showed a considerably increased activity in both renal veins (fig 8). At operation the abdomen was first opened and the abdominal aorta and its branches were dissected out. Both the renal arteries and the interrenal aorta were unaffected, though they were grossly distorted with a narrow calibre resulting from the low flow. The chest was then opened, and a Dacron graft was inserted between the upper end of the descending aorta and the abdominal aorta just above the origin of the inferior mesenteric artery. Another graft was inserted between the right common iliac artery and the superior mesenteric artery about $10 \mathrm{~cm}$ distal to its origin. The patient made an uneventful recovery, her arterial pressure has remained normal, and her body weight has increased to normal levels. Postoperative radioisotope renography showed normal renal blood flow (fig 8), and postoperative angiography (fig 9) showed normal circulation in the abdominal aorta and its branches.

Every patient in this series was investigated for evidence of active or healed tuberculous lesions both before and during operation, but these were never found. The results of surgical treatment are summarised in the table. The arterial pressure fell immediately after operation to low normal levels. This was followed by a slight rise in the late postoperative period and then a slow gradual fall. The postoperative pressures noted in this table are stable pressures over a follow-up period of more than one year. So far the patients available for follow-up have not shown any extension or recurrence of the disease, nor have they been kept on any long-term drug treatment.

\section{Technique of operation}

The principle of bypass grafting followed in this series was to anastomose both ends of the graft well beyond the area of disease. All the cases needed thoracoabdominal bypass grafts. Since the lesions in the descending thoracic and thoracoabdominal aorta were diffuse, the ends of the grafts usually had to be anastomosed close to the arch of the aorta above and just proximal to the bifurcation of the abdominal aorta below. A single 


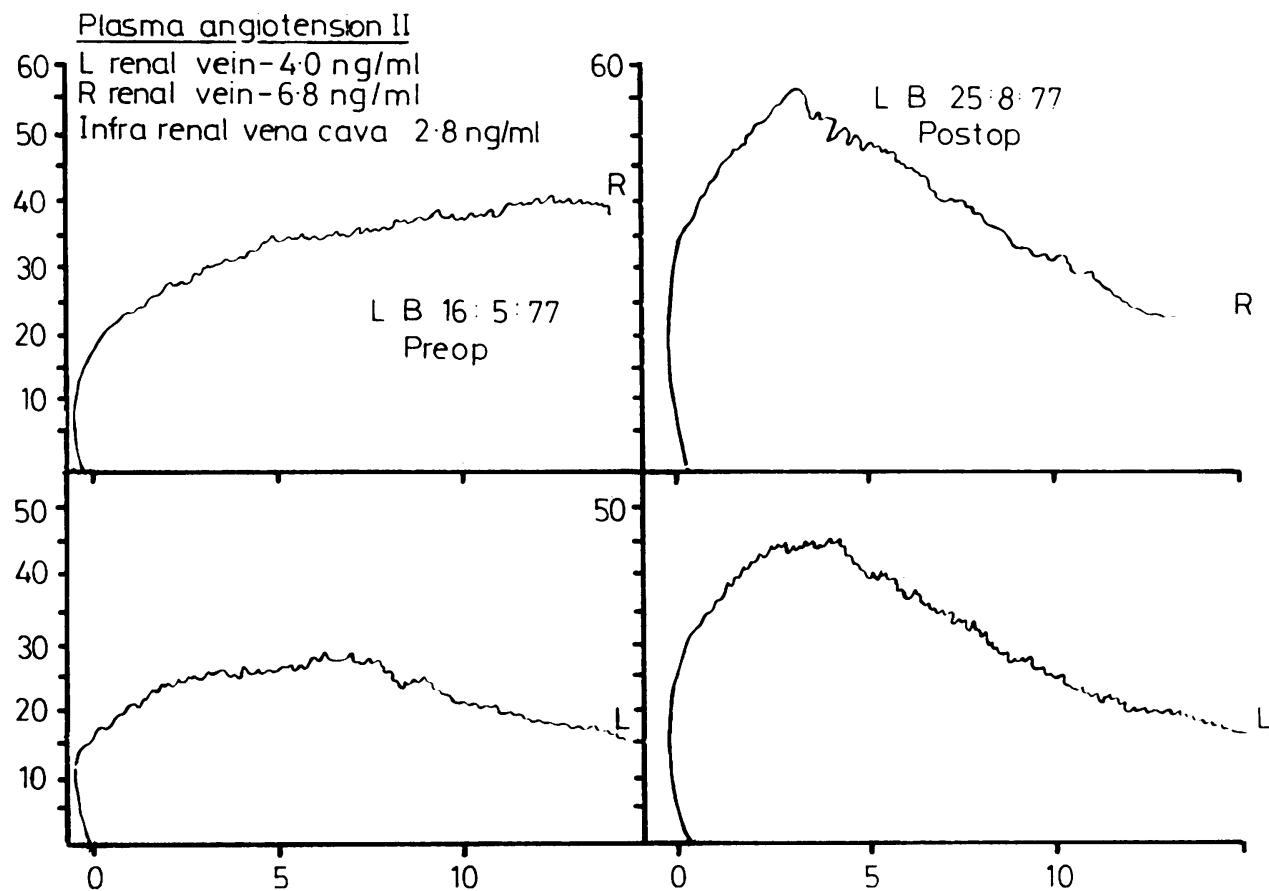

Fig 8 Case 11. Preoperative (left half) and postoperative (right half) radioisotope renogram and preoperative angiotensin assay. $R=$ Right kidney. $L=$ Left kidney. $x$ axis is time in minutes after injection of ${ }^{13} I$ Hippuran. $y$ axis is radioactivity (counts/min).

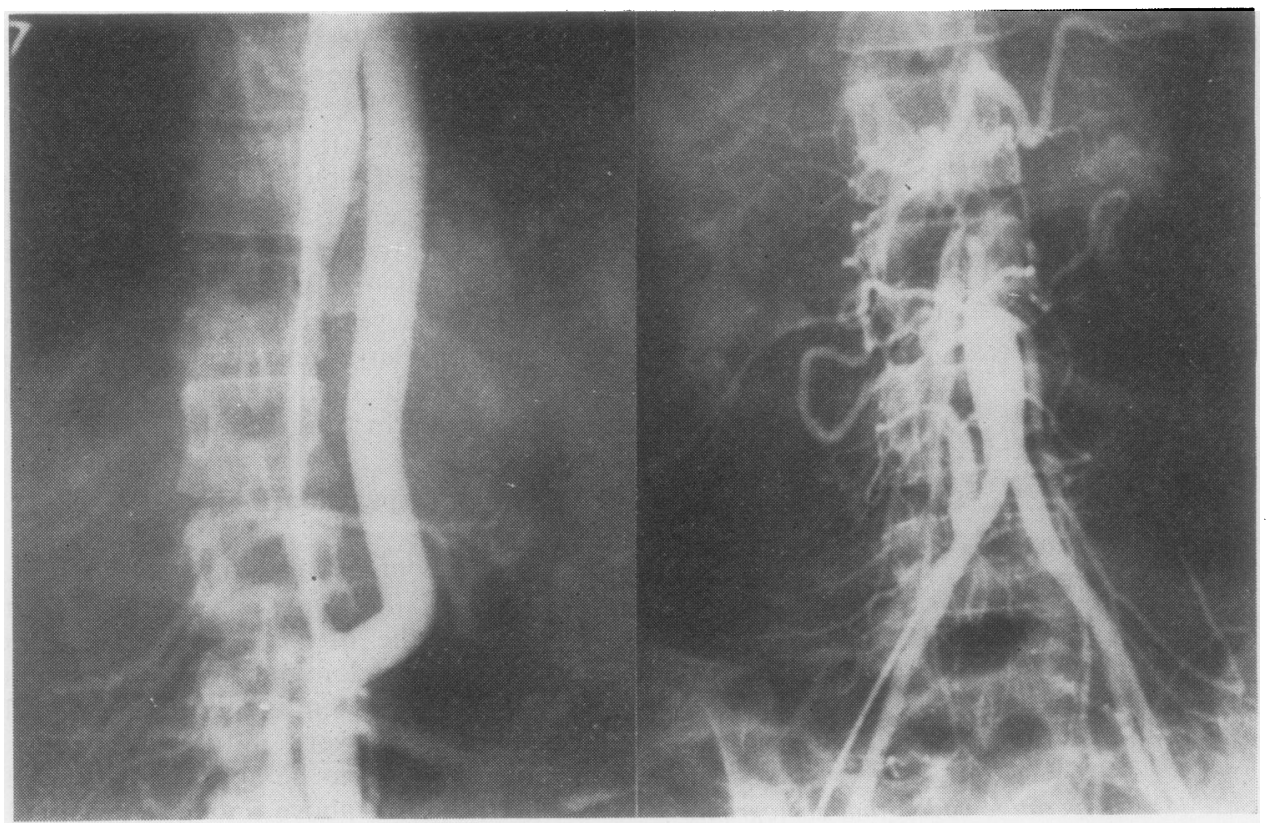

Fig 9 Case 11. Postoperative abdominal aortogram. (See text for description.) 
Results of surgical treatment. Total number of patients (11), surgical repair (seven), and survived (five)

\begin{tabular}{lll}
\hline $\begin{array}{l}\text { Case } \\
\text { No }\end{array}$ & $\begin{array}{l}\text { Preoperative } \\
\text { arterial pressure } \\
(\text { mmHg })\end{array}$ & $\begin{array}{l}\text { Postoperative } \\
\text { arterial pressure } \\
(\text { mmHg })\end{array}$ \\
\hline 5 & $170 / 110$ & $?$ \\
6 & $200 / 100$ & $140 / 90$ \\
7 & $190 / 110$ & $130 / 80$ \\
10 & $230 / 100$ & $110 / 60$ \\
11 & $190 / 110$ & $130 / 80$ \\
\hline
\end{tabular}

thoracoabdominal incision (Hallman et al, 1966) was unsuitable in most cases. With the patient in an oblique position, the incisions shown in fig 10 were made to open the chest and abdomen separately. Our present policy is to open the abdomen first to dissect out the abdominal aorta and its branches before proceeding further. Dissection of the right renal artery was somewhat difficult in this position but could be achieved by a lateral tilt of the operating table. The graft was passed from the chest to the abdomen through the paravertebral gutter by lifting the attachments of the diaphragm and then by tunnelling in the retroperitoneal tissues behind the pancreas and in front of the renal pedicle. The secondary graft in case 11 of this series was attached to the right common iliac artery because with this exposure it was difficult to attach a secondary graft to the aortic bypass graft after it was placed in its tunnel. The size of the main aortic graft was usually slightly smaller than the dilated proximal aorta but much larger than the attenuated abdominal aorta.

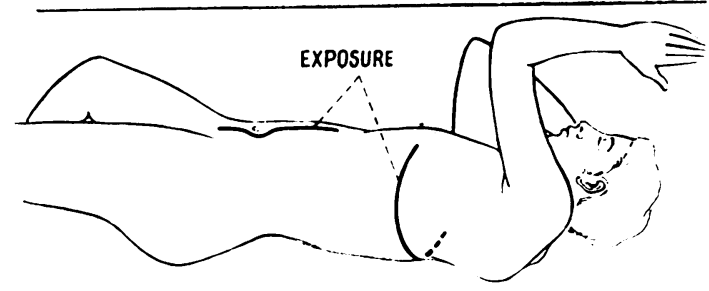

Fig 10 The position of patient and skin incisions for exposure of chest and abdomen for a long thoracoabdominal aortic bypass graft.

\section{Discussion}

The surgical treatment of non-specific aortitis is complicated by two characteristics of the disease. They are, firstly, the diffuse and extensive nature of the lesions and, secondly, lack of accurate knowledge regarding geographical distribution of the preferred sites and extent of affection as in the case of atherosclerosis. There is, however, one redeeming feature compared with atherosclerosis. The patients are younger, and their vascular tree beyond the level of obstruction is usually normal. The common practice is to decide on the feasibility of surgical treatment mainly on the basis of angiographic evidence. We have found that in some cases surgical exploration gives a better idea of the extent of disease and planning of surgical correction. There are examples of such experience in this small series, and it is possible to comment despite the small numbers.

Occlusion of the coeliac and superior mesenteric arteries in cases 9 and 11 was not associated with any symptoms of intestinal ischaemia, though when left uncorrected, as in case 9, it resulted in intestinal gangrene. Possibly a narrow lumen is kept open by a high head of pressure allowing a small flow of blood, but with the fall of pressure after insertion of the aortic bypass graft even this small flow is cut off. Angiography in the lateral projection is usually considered a better method of diagnosing obstructions to the coeliac and superior mesenteric arteries, but the possibility of skip lesions as in case 9 makes an anteroposterior angiogram followed by surgical exploration an adequate method of diagnosis.

Organic involvement of the renal arteries in the fibrotic process was also difficult to assess before operation. Distortion and haemodynamic alterations in the aorta distal to the site of obstruction produced an angiographic picture simulating involvement of the renal arteries and inter-renal aorta in case 11. Radioisotope renography in cases 7,10 , and 11 showed bilateral renal ischaemia. This was confirmed by angiotensin estimation in the renal veins in case 11 . But in cases 10 and 11 there was no renal artery stenosis, and in case 7 only the left renal artery was compressed from outside by a fibrotic collar at its origin.

The lower abdominal aorta below the stricture was not opacified in case 9, and in almost all cases it was narrow. This was the result of low flow and was not caused by any intrinsic disease. In the postoperative angiographic pictures of cases 7 and 11 this part has regained its normal calibre.

All the stenosed branches of the abdominal aorta should be dealt with to achieve satisfactory results after surgical correction. Intraluminal pressure measurement by direct puncture during operation is a useful method in detecting branch stenosis and has been used occasionally. Valid measurements can be obtained only after insertion 
of the main aortic graft when the aortic pressures above and below the stricture are equalised and retrograde perfusion of the branches below the stricture is possible.

Active or healed tuberculous lesions were conspicuously absent in all our cases, unlike those reported by Sen et al (1962). The maximum lesion in most cases was located at the junction of the thoracic and abdominal aorta at or close to the origins of the coeliac and superior mesenteric arteries, an area normally surrounded by intestinal lymphatics. This may suggest that the aetiology of the condition is related to an immune reaction to the parasitic infestations of the intestine that are extremely common in the socioeconomic groups usually affected by this disease. Possibly several different aetiological agents can produce the same histological picture through the same type of immune reaction (BMJ, 1977) in the aorta and other arteries.

Surgical correction has succeeded in roughly two-thirds of the cases of acquired coarctation of the aorta in this series. Possibly if a policy of routine surgical exploration is adopted when treating this disease a higher proportion of cases may be found to be correctable in future. Such exploration is justified in view of the excellent results expected in these young patients after total correction of the defects. Relief from hypertension after surgical correction can be as good as that after surgical correction of congenital coarctation of the aorta (Gupta et al, 1978).

I thank Professor C C Mallik, principal, NRS Medical College, Calcutta, for permission to use hospital records and publish the paper, Professor S R Mukherjee, professor-director (retired), Department of Experimental Medicine, Institute of Postgraduate Medical Education and Research, Calcutta, for the radioisotope renograms, and Professor A K Chaudhuri, professor of medicine, NRS Medical College, for arranging the angiotensin assay.

\section{References}

Bahnson, H T, Cooley, R N, and Sloan, R D (1949). Coarctation of the aorta at unusual sites. American $\widetilde{\Phi}$ Heart Journal, 38, 905-913.

Basu, A K (1961). Occlusive disease of the aorta and कs its main branches. British Journal of Surgery, 49, $\vec{\circ}$ 148-156.

British Medical Journal (1977). Takayasu's arteritis, $\vec{\omega}$ $1,667$.

Broadbent, W H (1875). Absence of pulsation in both $\overrightarrow{\vec{x}}$ radial arteries, vessels being full of blood. Tran- $\dot{\omega}$ sactions of the Clinical Society of London, 8, 165 168.

DeBakey, M E, Morris, G C, Jordan, G L, and Cooley, D A (1958). Segmental thrombo-obliterative $ᄋ$ disease of branches of aortic arch. Successful surgical treatment. Journal of the American Medical $\overrightarrow{ }$ Association, 166, 998-1003.

Gupta, S, Goswami, B, Sarkar, S, and Saha, $\mathbf{P} \mathrm{K}$ (1978). Congenital and acquired coarctation of $\stackrel{\infty}{+}$ aorta. Journal of the Indian Medical Association, $\vec{\theta}$ 70, 148-151.

Hallman, G L, Bloodwell, R D, and Cooley, D A (1966). Coarctation of the thoracic aorta. Surgical Clinics of North America, 46, 893-899.

Konar, N R, Chaudhury, D C R, and Basu, A K o (1955). A case of coarctation of aorta at an unusual site. American Heart Journal, 49, 275.

Regoli, D, and Vane, J R (1964). A sensitive method for the assay of angiotensin. British Journal of Pharmacology, 23, 351-359.

Sen, P K, Kinare, S G, Kulkarni, T P, and Parulkar, G B (1962). Stenosing aortitis of unknown aetiology. Surgery, 51, 317-325.

Sen, P K, Kinare, S G, Engineer, S D, and Parulkar, G B (1963). The middle aortic syndrome. British Heart Journal, 25, 610-618.

Shikhare, P V (1921). Notes on a remarkable case of absence of pulsation in the arteries of the upper parts of the body. Indian Journal of Medicine, 2, 326.

Takayasu, M (1908). Case of queer changes in central blood vessels of retina. Acta Societatis Ophthalmological Japonical, 12, 554.

Requests for reprints to: Dr S Gupta, 4 Bishop Lefroy Road, Calcutta 700020 . 